\title{
Razões que levam os pacientes com tuberculose \\ a abandonarem o tratamento: perspectivas do enfermeiro
}

\section{Reasons that take patients with tuberculosis to discontinue treatment: the nurse's perspectives}

\author{
Mirian Adriana Sackser' • Anelise Miritz Borges²
}

\begin{abstract}
RESUMO
Objetivo: Identificar os motivos que levam os pacientes a desistir do tratamento da tuberculose no município de Santa Cruz do Sul, a partir da visão dos enfermeiros que atuam em unidades de atenção primária de saúde. Metodologia:Tratase de uma pesquisa descritiva, exploratória, com delineamento qualitativo, realizada em cinco Unidades Básicas de Saúde (UBS) e cinco Estratégias de Saúde da Família (ESF) vinculadas ao município de Santa Cruz do Sul/Rio Grande do Sul. Para a coleta de dados foi realizada entrevista, utilizando um questionário semiestruturado, gravado, aplicado individualmente. A Análise foi conduzida pela análise de Conteúdo, temática de Bardin 20I I. Principais resultados: Foram 10 enfermeiras entrevistadas, metade possuía menos de um ano de experiência na unidade, referiram dificuldades no manejo dos sintomáticos respiratórios como a falta de tempo, recursos humanos e dificuldades de envolver o usuário no tratamento. $\mathrm{Na}$ perspectiva das enfermeiras entre os motivos envolvidos no abandono, estão o tempo terapêutico elevado, fatores sociais, e os efeitos colaterais dos medicamentos. Conclusão:A qualificação contínua da equipe sobre o manejo frente a doença, fortalece a assistência em saúde, tornando o usuário mais seguro quanto ao seu autocuidado, reduzindo as chances de abandono do tratamento.
\end{abstract}

Descritores: Tuberculose; Enfermagem; Saúde coletiva; Pacientes desistentes do tratamento

\section{ABSTRACT}

Objective: To identify the reasons that lead patients to give up tuberculosis treatment in the municipality of Santa Cruz do Sul, based on the view of nurses working in primary health care units. Methodology: This is a descriptive, exploratory, qualitative study, carried out in five Basic Health Units (UBS in Portuguese) and five Family Health Strategies (ESF in Portuguese) linked to the municipality of Santa Cruz do Sul/Rio Grande do Sul. For the data collection, an interview was conducted using a semi-structured, recorded questionnaire, applied individually. The analysis was conducted by the analysis of the thematic content of Bardin 20II. Main results:Ten nurses were interviewed and half of them had less than one year of experience in the unit, they reported difficulties in the management of respiratory symptoms such as lack of time, human resources and difficulties to involve the user in the treatment. From the perspective of nurses between the reasons involved in the abandonment, are the high therapeutic time, social factors, and the side effects of the medications. Conclusion: Continuous qualification of staff on the front handling the disease, strengthens health care, making the user more secure about their self-care, reducing the chances of noncompliance.

Keywords: Tuberculosis; Nursing; Public Health; Patient Dropouts

'Enfermeira. Graduada pelo Curso de Enfermagem junto à Universidade de Santa Cruz do Sul (UNISC). Atua no Hospital São Sebastião Mártir, Unidade de Terapia Intensiva, no município de Venâncio Aires, Rio Grande do Sul (RS).Email: amiritz@unisc.br

${ }^{2}$ Enfermeira. Mestre em Ciências da Saúde pela Escola de Enfermagem junto à Universidade Federal de Pelotas (UFPel). Doutora em Enfermagem pela Escola de Enfermagem junto à Universidade Federal do Rio Grande (FURG). Professora do Departamento de Enfermagem e Odontologia da Universidade de Santa Cruz do Sul (UNISC), Rio Grande do Sul (RS). 


\section{INTRODUÇÃO}

A Tuberculose (TB) é uma patologia infecciosa, causada pela bactéria Mycobacterium tuberculosis, essa doença pode afetar o pulmão, sendo denominada de TB pulmonar, ou afetar outros órgãos e ser chamada de TB extrapulmonar, sendo essa última menos frequente. É uma doença que possui tratamento, desde que o paciente siga o esquema de medicações e o tempo correto'.

A TB é considerada um problema global de saúde pública devido à sua alta prevalência em muitos países, dentre eles o Brasil, está associada as condições socioeconômicas da população, assim como aos problemas relacionados com a prevenção, o diagnóstico, o tratamento e a qualidade dos serviços de saúde ${ }^{2}$. O Brasil faz parte do grupo dos 22 países priorizados pela Organização Mundial da Saúde, que concentram $80 \%$ dos casos de TB notificados no mundo, ocupando a $16^{a}$ posição em número absoluto de casos ${ }^{3}$.

O Estado do Rio Grande do Sul se destaca com os maiores índices de casos de pacientes em retratamento de TB, logo, ao direcionar a percepção à Santa Cruz do Sul, constata-se que a cidade se encontra em alerta, pois faz parte dos 15 municípios prioritários para o controle da TB no Rio Grande do Sul, perante ao Plano Nacional de Controle da Tuberculose (PNCT) ${ }^{6}$.

No que se refere ao abandono do tratamento, esse se constitui como um dos principais desafios para o sistema de saúde brasileiro, e para os profissionais da saúde, é caracterizado pelo não comparecimento do usuário ao serviço de saúde por mais de trinta dias consecutivos, após a data marcada para a próxima consulta ${ }^{4}$. $\mathrm{O}$ abandono do tratamento da TB pode ser observado em grande proporção quando as ações de prevenção não são planejadas e realizadas adequadamente ${ }^{2}$.

O enfermeiro possui relevância na minimização dos casos de abandono do tratamento, sendo necessário que o mesmo se aproxime dos reais motivos que levam os usuários acometidos por TB a desistirem do tratamento. Esta ação permite que as orientações em saúde, sejam condizentes com o plano de assistência individual e com a realidade social do usuário, a fim de que ele assuma conjuntamente, responsabilidades no tratamento. Logo, tem-se como objetivo identificar os motivos que levam os pacientes a desistir do tratamento da tuberculose no município de Santa Cruz do Sul (SCS), a partir da visão dos enfermeiros que atuam em unidades de Atenção Primária de Saúde (APS).

\section{METODOLOGIA}

Trata-se de uma pesquisa exploratória e descritiva, com delineamento qualitativo conduzido no município de SCS, o qual possui uma população aproximada de I I8.374 habitantes, localiza-se no nordeste do estado do Rio Grande do Sul, a 155 km de sua capital, Porto Alegre. Possui área de $733 \mathrm{~km}^{2}$ e é referência econômica regional da região do Vale do Rio Pardo ${ }^{6}$, e contempla uma Unidade de Referência Municipal para Tratamento da TB (URTB), localizada na Unidade Acadêmica do Hospital Santa Cruz (HSC), em que os casos positivos são referenciados pela APS para a unidade referência, onde encontra-se profissionais especializados no tratamento da $\mathrm{TB}^{6}$.

A pesquisa foi realizada em cinco Unidades Básicas de Saúde (UBS): Avenida, Esmeralda, Verena, Jacob Schmidt e Farroupilha. E em cinco Estratégias de Saúde da Família (ESF): Gaspar Bartholomay, Faxinal, Cristal/Harmonia, Margarida e Viver Bem, todas estas unidades, localizadas na região urbana do município de SCS.A definição destes locais de pesquisa ocorreu a partir do levantamento do maior número de casos notificados no ano de 2016, obtidos junto à Vigilância Epidemiológica de SCS.

Fizeram parte da amostra deste estudo, 10 enfermeiras que atuavam nas unidades referidas acima, logo, como critério de inclusão tiveram-se: ser enfermeira responsável pela unidade. Para a coleta de dados foi realizada entrevista utilizando um questionário semiestruturado, gravado, aplicado individualmente pela discente nos locais de atuação das participantes, em dias previamente agendados. Quanto à identificação das enfermeiras, esta foi realizada utilizando o número do questionário, conforme a ordem de aplicação dos mesmos.

Os dados obtidos foram transcritos apoiando-se no Microsoft Word 2013, e os resultados analisados por meio da análise de conteúdo por temas ${ }^{5}$, a qual possui três momentos metodológicos: o primeiro momento consiste na pré-análise; o segundo na exploração do material, e o terceiro no tratamento e interpretação dos resultados. Assim, para a apresentação da análise foram definidas quatro temáticas, as quais são: Orientações sobre a tuberculose: o preparo do enfermeiro e do ACS; Atuação do enfermeiro: rotinas, fluxos e mobilizações na prevenção da tuberculose; Usuários suspeitos e assistidos com tuberculose: e o abandono e Dificuldades do enfermeiro e dos usuários no enfrentamento do controle e abandono do tratamento da tuberculose.

As exigências éticas e científicas foram preconizadas na pesquisa com seres humanos, fundamentando-se nos princípios éticos previstos na Resolução 466/20I2, do Conselho Nacional de Saúde. Obteve-se também, a autorização da Secretaria Municipal de Saúde e aprovação do Comitê de Ética em Pesquisa da Universidade de SCS, sob protocolo $n^{\circ} 2.190 .879$. Foram fornecidos esclarecimentos sobre o trabalho e assegurado a responsabilidade ética por meio do Termo de Consentimento Livre e Esclarecido às participantes. 


\section{RESULTADOS}

Participaram da pesquisa 10 enfermeiras atuantes em unidades de APS do município de SCS, cinco delas trabaIhavam em UBS, possuíam idades entre 29 e 42 anos e quatro delas, tinham pós-graduação na saúde pública, já quanto ao tempo mínimo de atuação no serviço, este foi de um mês a três anos. Nas ESF, as idades variaram entre 30 a 61 anos, quatro possuíam pós-graduação na saúde pública e quanto ao tempo mínimo de atuação, este variou entre nove meses a 13 anos. Todos os resultados podem ser verificados na tabela I a seguir:

\section{Orientações sobre a tuberculose: o preparo do enfermeiro e dos agentes comunitários de saúde}

Ao questionar as enfermeiras a respeito da sua participação em treinamentos ou capacitações sobre a tuberculose, nove responderam afirmativamente, detalhando os conteúdos abordados, como podemos ver a seguir:

Sim, orientações sobre a identificação dos sintomáticos respiratórios, orientar sobre a coleta, cuidados em casa com os demais familiares, sobre o tratamento que não é mais como antigamente, diminuiu o número de comprimidos, os medicamentos não estão mais tão agressivos, da importância de tomar a medicação e fazer os exames de controle, os medicamentos estão mais eficazes. Mesmo sendo uma doença bem antiga, ela existe e tem tabus [...]. (04)

Sim, como orientar o paciente, a busca ativa dos sintomáticos respiratórios, toda a equipe precisa estar atenta. Como fazer a coleta, aonde fazer essa coleta, a primeira pode fazer em qualquer horário do dia e a segunda tem que ser de manhã em jejum [...]. (06) Sim, sobre a coleta, busca ativa dos sintomáticos respiratórios, tratamento, notificação [...]. (I0)

Quanto às instituições que viabilizaram as orientações sobre a tuberculose, quatro participantes referiram ser a enfermeira vinculada à Secretaria Municipal de Saúde, esta responsável pelo programa. Duas citaram ser fornecido pela Secretaria Municipal de Saúde e outras duas participantes responderam ser a $13^{\mathrm{a}}$ Coordenadoria Regional de Saúde. Uma enfermeira não lembrava quem havia promovido o treinamento e outra não recebeu capacitação.

Ressalta-se que mesmo diante das capacitações recebidas, duas enfermeiras manifestaram dificuldades na efetividade do que foi orientado, pois o fluxo desde a coleta do escarro até a obtenção de um tratamento eficiente,

TABELA 1 - Perfil das enfermeiras atuantes em 10 unidades de atenção primária em saúde de Santa Cruz do Sul, Rio Grande do Sul, 2017.

\begin{tabular}{|c|c|c|}
\hline \multirow[t]{2}{*}{ Variáveis } & $\begin{array}{c}\text { Unidade Básica } \\
\text { de Saúde }\end{array}$ & $\begin{array}{c}\text { Estratégia Saúde } \\
\text { da Família }\end{array}$ \\
\hline & $\mathbf{n}$ & $\mathbf{n}$ \\
\hline \multicolumn{3}{|l|}{ Idade } \\
\hline 29-39 anos & 03 & 02 \\
\hline $40-50$ anos & 02 & 01 \\
\hline 51-61 anos & - & 02 \\
\hline \multicolumn{3}{|l|}{ Pós-graduação } \\
\hline \multicolumn{3}{|l|}{ Lato sensu } \\
\hline Saúde Pública & 02 & - \\
\hline Saúde da Família & 04 & 02 \\
\hline Saúde do Trabalhador & - & 02 \\
\hline Saúde da Pessoa Idosa & 02 & - \\
\hline Unidade de Terapia Intensiva & 01 & - \\
\hline Residência Multiprofissional & 01 & - \\
\hline Urgência/Emergência/Trauma & - & 01 \\
\hline Oncologia & 01 & - \\
\hline \multicolumn{3}{|l|}{ Stricto sensu } \\
\hline Mestrado Ciências da Saúde & 01 & - \\
\hline \multicolumn{3}{|l|}{ Tempo atuação } \\
\hline$<1$ anos & 03 & 02 \\
\hline$>1 \leq 5$ anos & 02 & - \\
\hline$>5 \leq 10$ anos & - & 01 \\
\hline$>10 \leq 15$ anos & - & 02 \\
\hline
\end{tabular}

Fonte: dados da pesquisa. 
requer uma infraestrutura mais adaptada ao contexto e demandas do município, o que pode contribuir para a subnotificação dos casos e possível aumento de pessoas com tuberculose, resultado verificado na fala a seguir:

A gente possui na unidade, o pote, só que a nossa grande dificuldade, na maioria das vezes, é o paciente ir fazer o exame. Então a gente orienta a coletar a primeira amostra na unidade, orienta ele da segunda amostra, mas muitas vezes ele não retorna com o exame, porque ele não coleta a segunda amostra e não leva para o laboratório. $E$ nós não temos aqui, ainda um local, pois como ele mora perto, poderia trazer a segunda amostra aqui e nos estarmos encaminhando. Isso está sendo visto pela coordenadora, para ter um frigobar na própria unidade, [...] um fluxo, uma logística de recolhimento toda sexta feira, assim como acontece com outros exames. Como tem que ser guardado em local apropriado e refrigerado, a gente não possui esse local apropriado [...]. (02)

A primeira coleta pode fazer em qualquer horário do dia e a segunda tem que ser de manhã em jejum. Na unidade não se tem um local apropriado [...]. (06)

Ainda sobre os treinamentos e capacitações recebidas, pode-se perceber que oito das enfermeiras avaliaram que os Agentes Comunitários de Saúde possuem preparo para participar na divulgação de orientações sobre a prevenção e tratamento da tuberculose em suas micro áreas, o que denota um fortalecimento no processo de educação em saúde in loco nos domicílios.

Sim, [...] porque muitas vezes o paciente não procura a unidade para uma consulta médica ou para dizer seus sinais e sintomas, mais o ACS estando dentro da casa do paciente, na visita de rotina, ele pode verificar que o paciente está apresentando tosse, relatando emagrecimento, suor noturno, ele está atendo e também pode estar referenciando e solicitando o exame. O ACS é o elo entre a unidade e o paciente [...]. (02) Sim, ações para o ACS tem bastante, as vezes a gente nota que falta para o resto da equipe, o ACS está lá na ponta, são profissionais relevantes, eles estão bem capacitados, tem um olhar de identificação dos casos, e eles que muitas vezes nos trazem demandas, fazem orientações aos pacientes, porque a gente está dentro da unidade e não consegue ver todos os pacientes [...]. (03) Sim, eles (ACS) estão bem sensibilizados para questão dos sintomáticos respiratórios e da busca ativa [...]. (I0)

Outro aspecto relevante quanto ao conhecimento dos ACS sobre a tuberculose incide na formação atualizada do enfermeiro da unidade, pois é ele a referência no esclarecimento de dúvidas. Muitas vezes, diante da demanda de tarefas na unidade ou até mesmo, a falta de recursos humanos na equipe, o enfermeiro não consegue participar de todas as capacitações ofertadas.
Os ACS possuem bastante duvidas [...] me questionam bastante e recebem capacitações da Enfermeira da Secretaria Municipal de Saúde [...]. (04)

OACS está preparado, eles têm mais capacitações que o próprio enfermeiro, nós acabamos ficando presos na unidade, por falta de pessoal [...]. (07)

Um enfermeiro relatou que em decorrência da substituição dos ACS contratados pelos concursados, o grupo de agentes necessitaria de investimentos voltados à educação em saúde sobre a tuberculose, para assim qualificar a assistência prestada aos usuários.

Nesse momento aqui mudou a equipe, e teria que ter capacitações, faz uns cinco meses que mudou toda a equipe dos ACS em função do concurso [...]. (06)

Diante da temática capacitação, quanto maior o preparo dos enfermeiros e ACS sobre a tuberculose, maiores serão as chances de reduzir o impacto da doença, haja visto que estar capacitado fortalece a assistência em saúde e a possibilidade de que, o aprendido pela comunidade, seja multiplicado a favor da prevenção da doença. $E$ diante de algumas dificuldades apontadas, o ato de refletir sobre o melhor a ser feito é algo dialogado entre as enfermeiras e a sua coordenação.

\section{Atuação do enfermeiro: rotinas, fluxos e mobili- zações na prevenção do abandono do tratamen- to da tuberculose}

Sobre a atuação do enfermeiro na unidade, diante da sistematização do trabalho com usuários em suspeita ou notificação de tuberculose, verificou-se que existe uma rotina para sete enfermeiras. Contudo, mesmo diante de todo o envolvimento dos integrantes da equipe, na obtenção do possível diagnóstico, alguns usuários não retornam com o resultado à unidade, o que pode ser identificado nas falas a seguir:

Sim, quando há suspeita de paciente com TB e estão apresentando tosse há mais de duas ou três semanas, febre vespertina ou não, quando há algum sinal suspeito, aí o médico solicita que a gente faça as orientações para a coleta do escarro, então a gente orienta como fazer a coleta da primeira amostra e da segunda amostra. A gente lança no livro, sede os frascos já denominados, primeira amostra e segunda amostra, com o nome dos pacientes e já manda junto com a autorização, com o nome da unidade, do paciente bem direitinho, que já vai com os laboratórios de referência. E orienta o paciente como coletar e como levar essas amostras, e a gente pede que esse paciente volte com o resultado, e esse é o problema, a maioria não volta com o resultado [...]. (0I)

Sim, tanto em consulta de enfermagem como em consulta médica, a gente sempre tem esse olhar dos sintomáticos respiratórios e já solicita exames, e tam- 
bém a gente sempre vai relembrando com as ACS [...] para que elas estejam atentas nas casas [...] a gente orienta que elas peçam ou mandem o paciente até $a$ unidade [...]. (03)

Dentre as rotinas, também foi referido por nove enfermeiras, a sua confiança frente ao trabalho das ACS, as quais são importantes quanto à sua atuação sobre a temática e possuem respaldo profissional para atuarem junto a linha de frente, na orientação dos usuários com possível suspeita de tuberculose.

As próprias ACS podem solicitar o exame, tem o formulário específico, elas podem entregar os potinhos, dar as orientações, mais é dificil, porque dependendo de como for feita essa abordagem, ou o nivel de compreensão do paciente, ele pode se apavorar, então sempre peço para elas pedirem para o paciente vir até a unidade, mais elas podem dar essas orientações [...]. (04)

As ACS têm um kit que a Coordenação do Programa fez, então elas mesmas já podem encaminhar. Esses três pacientes que eu te citei, dois já ganharam alta, só estamos com um ainda em tratamento [...]. (09)

Foram três enfermeiras, que referiram não apresentar uma rotina/sistematização diante de usuários sintomáticos respiratórios, fato relacionado com a não priorização desta conduta ou até mesmo com a inserção de ações dentro da rotina diária da equipe, sem que haja enfoque específico.

$O$ que a gente fazia era anotar um telefone e fazer uma ligação para ver, mas o que constatamos, é que eles dizem que não fizeram o exame, então não vai ter resultado [...]. (02)

Ainda não, preciso organizar essas ações [...]. (05)

Não. Mas se na visita domiciliar o ACS perceber uma paciente com muita tosse, persistente, ele vai encaminhar para unidade. E a gente vai facilitar o acesso desse paciente na consulta. Está incluído como demandas da unidade, mais não tem um trabalho especifico para isso [...]. (06)

Ao questionar as enfermeiras como elas realizavam as orientações da coleta para os usuários, pode-se verificar o cuidado quanto ao acolhimento, o detalhamento e o discernimento frente à equidade desde a primeira consulta na unidade, para que assim ocorra a confiança no serviço prestado e o êxito na ação almejada. Quanto ao fluxo, sete enfermeiras relataram orientar o usuário a estar em jejum para coletar a segunda amostra.

Geralmente eu oriento que faça em casa, mais se eu observar que é uma pessoa bem vulnerável, eu já faço a primeira coleta na unidade, o ideal é levantar da cama e fazer a coleta, eu oriento a não comer nada, não escovar os dentes antes de fazer a coleta. Oriento que não pode ser saliva, tem que ser escarro [...]. (04)

A gente sempre orienta a fazer a coleta em jejum, são dois dias consecutivos de coleta, na primeira hora da manhã, que seja um ambiente ventilado e sem fluxo de pessoas, que inspire fundo algumas vezes antes da coleta, que consiga o máximo de escarro possível [...]. (10)

Sobre o tempo que o exame de escarro leva para a obtenção do resultado, três enfermeiras responderam que este é de uma semana a 10 dias, contudo uma destas enfermeiras orienta para que o usuário contate o laboratório, a fim de obter a resposta. Duas enfermeiras responderam que leva entre dois ou três dias, outras duas falaram que leva em torno de um mês a 45 dias, e outras três enfermeiras não souberam responder.

Acredito que leva em torno de 10 dias para sair o resultado do exame. Quando o paciente está muito ansioso, peço que ele questione o laboratório sobre $o$ tempo que leva para sair o resultado [...]. (04)

Em torno de uns 45 dias acho que o exame fica pronto [...]. (05)

O tempo que leva para ficar pronto eu não sei dizer [...]. (OI)

Quando o paciente retorna à unidade com o resultado do exame de escarro, a rotina adotada por nove enfermeiras foi entrar em contato com o ambulatório da tuberculose com intuito de encaminhar o paciente. Apenas uma enfermeira revelou que realizava o tratamento na unidade de atenção primária, não citando o ambulatório.

Se positivo, encaminhar para o ambulatório da TB, o paciente já sai da unidade APS, com a consulta agendada com o médico infectologista [...]. (02)

Orientar a fazer o uso dos medicamentos corretamente, não abandonar o tratamento, se é um paciente bem ciente, pode fazer o acompanhamento pelo posto, mais ele precisa ir até o ambulatório pegar a medicação, já acompanhei pacientes que precisavam vir até $a$ unidade para nos observarmos ele tomar a medicação [...]. (08).

Assim, é muito importante o usuário com TB, sair da unidade de APS bem orientado, com o encaminhamento devido ao ambulatório de referência, tornando evidente, neste processo, a necessidade do diálogo constante entre os profissionais destes serviços, a fim de identificar possíveis falhas na adesão ao tratamento e iniciativas para a busca ativa.

\section{Usuários suspeitos e assistidos com tuberculose: e o abandono?}

Sobre a notificação de casos de tuberculose, no ano de 2016, nas unidades alvos da pesquisa, pode-se constatar que oito pacientes foram diagnosticados com tuberculose, sendo os oito pacientes vinculados a três unidades de saúde, dados estes obtidos junto ao livro dos sintomáticos respiratórios.

Destaca-se que 66 pacientes sintomáticos respirató- 
rios foram encaminhados por oito unidades alvo da pesquisa para fazer o exame de escarro no ano de 2016, as outras duas unidades não tinham nenhum registro no livro dos sintomáticos respiratórios, e as enfermeiras não souberam responder, pois uma não atuava na unidade neste período e a outra não realizava o registro.

Referente aos oitos usuários com tuberculose no ano de 2016, as enfermeiras foram questionadas quanto ao abandono do tratamento medicamentoso, sendo afirmado a desistência de um usuário, o que pode ser visto na fala a seguir:

Sim, um paciente abandonou, teve que passar por internação em Porto Alegre no Hospital Paternon e quando ele voltou para Santa Cruz, ele terminou o tratamento [...]. (07)

Conforme apresentado nos resultados acima, apreende-se que é importante viabilizar orientações de forma clara e segura ao usuário, buscando responsabilizá-lo de forma consciente do autocuidado com a sua saúde.

\section{Dificuldades do enfermeiro e usuários no en- frentamento do controle e abandono do trata- mento da tuberculose}

Frente às dificuldades do dia-a-dia das enfermeiras no manejo do (LSR) nove delas afirmaram não apresentar dúvidas no preenchimento do livro, contudo uma delas afirmou que a grande demanda de atividades na unidade, por vezes atrapalha o registro dos casos. Outra enfermeira referiu que os médicos poderiam participar deste trabalho.

Não se tem dificuldades, o que acontece ás vezes é a dificuldade de registrar, por causa das demandas. As vezes se deixa para depois e se acaba esquecendo [...]. (06)

A dificuldade é os médicos registrarem. Dificuldade em o paciente trazer o resultado para registrar no livro [...]. (07)

Ainda sobre as dificuldades, todas as enfermeiras manifestaram algum apontamento, como a falta de tempo, de recursos humanos e de sensibilizar o usuário para aderir às condutas profissionais. Somente para as UBS foi ressaltada a reduzida estrutura física e a unidade não possuir território delimitado, motivos dos quais, podem interferir positivamente nos casos de abandono.

Desgaste emocional que a gente tem quando tem um paciente que ele não quer fazer o tratamento, o maior interessado tinha que ser ele. (03)

Tem áreas que não possuem ACS, a área da UBS é muito grande, não conseguimos ter o controle de tudo [...]. (04)

Falta de pessoal, hoje estou com deficiência de técnicos, e acaba que ficamos presos a unidade e não conseguimos fazer as visitas como se deveria fazer [...]. (07)
Falta transporte para ir até o paciente e fazer essa busca ativa aos faltosos. Tempo também falta, porque a demanda é grande, você pode observar na minha mesa a quantidade de papeis que eu acabo não dando conta, isso que hoje deu uma trégua no movimento por causa do tempo [...]. (05)

Como é uma unidade aberta, não se tem uma população definida, uma pessoa pode vir aqui consultar e não ser morador do bairro, a gente descobrir e enfim não ter ACS, qualquer pessoa pode consultar aqui e você acaba não tendo aquele vinculo de conhecer melhor o paciente, saber onde ele mora, questão de estrutura também, porque não tem nem como fazer uma consulta de enfermagem com melhores orientações, eu não tenho uma sala para mim, esse espaço foi improvisado aqui para mim. Só tem um consultório médico, uma sala de vacina [...]. (08)

Em relação às dificuldades que as enfermeiras identificaram que o paciente com tuberculose apresentava para seguir o tratamento, seis enfermeiras referiram ser o tempo terapêutico elevado, assim como os fatores sociais, quanto ao uso de drogas, alcoolismo, falta de estrutura familiar e ser acamado. Também foi apontada a ação dos efeitos colaterais dos medicamentos como prejuízo ao corpo humano.

Uso de drogas, alcoolismo. Questões sociais desfavoráveis, familias desestruturadas [...]. (06)

Pacientes com alcoolismo, drogados. No caso de abandono na nossa unidade era a mãe e filho que usavam álcool, e o filho desistiu do tratamento [...]. (07)

Para nós a maior dificuldade são os fatores sociais, porque quase todos que apresentaram problemas no tratamento, eram sozinhos e acamados [...]. (09)

Eu acho que é o efeito da medicação, eles reclamam que no início apresentam muitos efeitos colaterais e passado um tempo eles abandonam, se eles não forem acompanhados e orientados logo quando inicia o tratamento acho que o abandono é muito grande [...]. (02)

Frente ao número de notificações de casos de tuberculose no ano de 2016 , pode-se constatar que dos oito pacientes em tratamento de TB e uma desistência, a maior dificuldade ainda recai na ausência do retorno à unidade com o resultado do escarro, pois muitos nem levam as amostras ao laboratório. Fato que denota uma falha em cadeia, em que o registro nos livros dos sintomáticos respiratórios, também deixa de ser efetivado devidamente.

\section{DISCUSSÃO}

Atuar na APS torna-se essencial, sob o ponto de vista da prevenção da disseminação da doença e manutenção da saúde, para tanto, visualiza-se no estudo ${ }^{7}$ realizado com I 4 enfermeiras atuantes em ESF, no Belém, Pará, que 
o enfermeiro é jovem, com idade entre 27 a 64anos. A maioria delas tinham feito curso de especialização, com destaque para a Saúde Pública ou Saúde da Família, mas também à área hospitalar. Em outro estudo realizado em ESF, no Rio Grande do Norte, a maioria pertencente a categoria profissional enfermeiro, era do sexo feminino, com idades que variavam entre 23 e 48 anos, atuando de três a quatro anos nas unidades 8 .

Assim, constata-se que o perfil identificado na presente pesquisa retrata também realidades de outras regiões, demonstrando que as enfermeiras são jovens e a maioria do sexo feminino, contudo frente ao tempo de atuação, este foi um pouco menor para as enfermeiras de SCS, pois metade delas possuía menos de um ano de experiência nas unidades.

O estudo realizado em Espirito Santo,Vitoria, demostrou que $50 \%$ dos enfermeiros, atuavam na equipe entre um a quatro anos, já sob a análise detalhada por estados, identificou-se que $\circ$ percentual de enfermeiros trabaIhando há menos de um ano em ESF e UBS variou desde o estado do Piauí, com I $3 \%$ e o Amapá, com $52 \%$. Não obstante, destaca-se para o Rio Grande do Sul, a taxa de 23\% e Santa Catarina, 32\%.

A rotatividade dos profissionais enfermeiros e demais membros da equipe, compromete o estabelecimento de vínculo com a população, assim como o planejamento e execução de ações em saúde, sejam elas conduzidas em ESF ou UBS. Outro aspecto relacionado com a rotatividade é a sobrecarga de trabalho para os profissionais que permanecem nas unidades, além de atrapalhar a organização e fluxos, os novos profissionais demandam treinamento por parte da equipe?.

Assim, desenvolver atividades em saúde que busquem orientar a população, requer do enfermeiro a compreensão de suas atribuições e dos dispositivos legais que regem a sua atuação nos serviços de atenção básica, sendo importante conhecer as políticas de saúde vigentes no País é estar atualizado quanto às demandas de sua unidade ${ }^{8}$, ação concernente também ao tema sobre tuberculose.

Uma das ferramentas importantes para o controle da TB são as capacitações destinadas aos profissionais da APS, as quais os auxiliam no êxito do planejamento, na detecção precoce dos casos e na qualidade da assistência prestada ${ }^{10}$. A oferta de cursos, oficinas e demais modalidades de treinamento, mantém os profissionais atualizados $^{8}$. Logo, possuir uma administração na APS, que disponibilize treinamentos à equipe profissional é um diferencial na presente pesquisa, o que denota um espaço para a construção contínua do conhecimento.

Em João Pessoa, um estudo conduzido com 10 enfermeiros de ESF demonstrou que este profissional é um ator importante para efetivação do cuidado da TB, pois gerencia as ações de controle frente a complexidade en- volvida nesse processo ". Contudo, em Teresina, no Piauí, os autores ${ }^{12}$, reforçam que o enfermeiro necessita qualificar a sua atuação no manejo de usuários SR, para assim viabilizar um cuidado integral e humanizado vinculado as ações do PNCT.

Quanto aos ACS, estes ganharam ainda mais destaque com a implantação das ESF, eles são o elo de comunicação entre a equipe de saúde e a população, por estarem inseridos na comunidade, desenvolvendo visitas domiciliares e orientações em saúde, permitindo viabilizar o repasse de suas ações para a equipe ${ }^{13}$. O ACS possui contato direto com os conhecimentos de educação popular, haja visto as atribuições do seu trabalho, o que o direciona também, a aliar estes dados obtidos na comunidade, com o conhecimento cientifico ${ }^{14}$.

Assim como ocorre em outras regiões, segundo as enfermeiras de SCS, o ACS desempenha um importante trabalho junto com a equipe das unidades, e se mostra cada vez mais empenhado na busca pelo conhecimento, para levar informações corretas à população.

Quanto à busca ativa feita pela equipe aos usuários SR, esta objetiva identificar precocemente pessoas com tosse igual ou superior há três semanas, e deve ser uma atividade priorizada nos serviços de saúde ${ }^{15}$. Portanto, fazer o diagnóstico da TB e iniciar de forma rápida o tratamento, são as principais estratégias para o controle da doença, logo, o planejamento das ações deve ser desenvolvido com enfoque na identificação do usuário SR, para tão logo, realizar o seu encaminhamento para obtenção do diagnóstico e o início do tratamento adequado ${ }^{15}$.

O PNCT orienta os municípios a oferecer o Tratamento Diretamente Observado (TDO), conhecido como Directly Observed Treatment Schort Course (DOTS) ${ }^{15}$. OTDO é um método definido pela equipe conjuntamente com o usuário, e consiste na forma de administrar os medicamentos, que é conduzida por um profissional treinado para observar o consumo de medicação feito pelo usuário, desde o início do tratamento até a sua cura. Esta ação requer a criação de um vínculo entre ambos no serviço de saúde, para remover as barreiras que impedem a adesão ao tratamento. Fato que requer a adoção de estratégias que visem a reabilitação social, melhora da autoestima e o convívio social ${ }^{15}$.

Em relação ao fluxo da coleta, diagnóstico e tratamento da TB, um estudo conduzido em São Paulo, revela dificuldades quanto ao fornecimento da assistência aos usuários $\mathrm{SR}^{16}$. Em um Centro de Saúde Escola gerenciado pela Universidade do Pará, o fluxo dos pacientes com TB se inicia a partir do acolhimento pelos técnicos de enfermagem, os quais solicitam duas amostras da baciloscopia de escarro. $O$ resultado é obtido em dois momentos, sendo a primeira amostra colhida na unidade e o seu resultado obtido em um dia, em média, e em dois dias, 
para a segunda, a qual é colhida na residência. Os autores ressaltam ainda, que diante dos resultados positivos na baciloscopia, os usuários SR são encaminhados para a consulta na sua unidade de referência, sendo que o tratamento se inicia no mesmo dia pela enfermeira. Para àqueles com baciloscopia negativa, mas com sintomas da $\mathrm{TB}$, o encaminhamento é feito para o pneumologista ${ }^{17}$.

Segundo o Ministério da Saúde, a coleta, a conservação e o transporte das amostras de escarro é responsabilidade dos serviços de saúde ${ }^{15}$. Porém, em SCS, as enfermeiras informaram que a unidade não possui um local apropriado para o armazenamento das amostras, ficando sob responsabilidade do usuário encaminhá-las até o laboratório. E quanto ao processo de coleta, relembra-se que em SCS, é o usuário que a realiza em sua residência e retira o resultado junto ao serviço, com um tempo aproximado de no mínimo dois a três dias, até no máximo 45 dias.

Quanto ao jejum, a maioria das enfermeiras de SCS citaram como uma importante orientação para o usuário, estar em jejum para coletar a segunda amostra no dia posterior a primeira. Sobre este aspecto, a pesquisa realizada em uma UBS, na Universidade do Pará, evidenciou que a unidade não possui um ambiente especifico para realizar as coletas, por isso, são orientados a coletar as amostras na residência, sendo a primeira coleta no dia seguinte ao atendimento, em jejum e a segunda no dia posterior a primeira ${ }^{17}$.

O recomendado é de que a primeira amostra seja coletada nas dependências da unidade, em um local arejado, para isso, o SR deve fazer a higiene das mãos e da cavidade oral sem utilizar creme dental ou soluções antissépticas, a segunda amostra pode ser coletada no domicilio, logo após acordar, estar em jejum, e seguir as mesmas recomendações da primeira amostra ${ }^{18}$. Quanto ao registro e acompanhamento dos casos, é utilizado o LSR, todas as unidades de APS devem possuí-lo devidamente preenchido a fim de possuir a identificação do SR para a efetivação do diagnóstico de TB e início do tratamento ${ }^{15}$. Permite também verificar o tempo decorrido entre a identificação do caso e a realização do exame pelo paciente, e observar o seguimento do protocolo que preconiza a coleta de duas amostras de escarro para - diagnóstico e ainda o índice de resultados positivos em cada unidade ${ }^{15}$. $O$ que pode dificultar o processo de identificação do SR devido à ausência do registro no LSR e/ou subnotificação dos casos de abandono, quando não descritos devidamente.

Um estudo realizado em Lima, Peru, com profissionais da saúde da APS, entre eles enfermeiros, destacouse que entre os aspectos relacionados ao abandono no tratamento, encontram-se as questões sociais, efeitos adversos das medicações, e os tabus que essa doença possui por ser contagiosa, motivos que levam as pessoas a se afastarem do portador, e este abandonar o tratamento ${ }^{19}$. Em um estudo realizado na cidade de São Paulo, os autores apontam que os motivos ligados a questão do abandono na APS estão relacionados a falta de capacitações para a equipe, demora nos resultados laboratoriais e agendamento de exames, ausência de profissionais que se dediquem exclusivamente para as ações do PNCT, falta de materiais, falta de um ambiente adequado para o acolhimento dos SR, bem com a ausência de um veículo próprio para os profissionais fazerem a busca ativa ${ }^{16}$.

O uso de drogas ou bebidas alcoólicas, está dentro das questões sociais citadas pelos profissionais como fatores relacionados ao abandono do tratamento da TB, em um estudo realizado em Rio Branco, no Acre, um enfermeiro de APS classificou que o tratamento de dependentes químicos, com TB é complicado, e destacou a necessidade de desenvolver estratégias para o manejo nesses casos específicos ${ }^{20}$. O usuário precisa ser assistido de forma integral, todos os esforços devem ser feitos com o objetivo de envolve-lo no seu próprio cuidado, assim como o fortalecimento do vínculo entre o usuário, profissional e serviço de saúde ${ }^{16}$.

Frente à visão de três portadores de TB quanto ao abandono dos seus tratamentos, uma pesquisa indicou o fato de obterem uma melhora do seu quadro clínico, antes mesmo de concluírem a terapia, assim como, a necessidade de ingerir bebidas alcoólicas, abdicando do tratamento ${ }^{20}$. Mas, também são evidenciados outros motivos, como os efeitos colaterais das medicações, a falta de atenção da equipe principalmente na questão dos encaminhamentos e informações básicas sobre a patologia ${ }^{2}$.

Logo, manter o vínculo com o usuário diagnosticado com TB, bem como à sua família, é uma ação essencial do enfermeiro, o qual necessita ousar do acolhimento, envolvendo toda a sua equipe da unidade, com vistas a articular estratégias que envolvam a redução de casos de abandono e a efetividade no tratamento.

\section{CONSIDERAÇÕES FINAIS}

Mesmo referindo receber capacitações sobre a temática, algumas vezes as enfermeiras não conseguiam efetivar as orientações na prática, pois dentre os obstáculos estavam a falta de tempo diante de muitas demandas na unidade, a mudança do quadro de funcionários frente à necessidade da constante construção de vínculo e necessidade de troca de saberes entre a equipe.

Outro aspecto salientado quanto às dificuldades está no espaço físico das unidades, que por vezes é pequeno e poderia conter um local específico para armazenar as amostras de escarro, ou até mesmo, haver um sistema de coleta destas culturas, semanalmente nos serviços de APS, reduzindo assim, os casos inconclusivos para a do- 
ença. Não obstante, a rotatividade de usuários nas unidades abertas, cuja população não é adscrita também se tornou um fator dificultador. Assim, todos estes fatores dificultam de alguma forma $\circ$ trabalho efetivo do enfermeiro e sua equipe na APS com os SR, contribuindo para que ocorram subnotificações da TB, diante de casos não registrados no LSR.

Quanto às dificuldades que os usuários com TB possuem para dar continuidade ao tratamento, pode-se constatar através das falas das enfermeiras que foram o tempo terapêutico elevado; os diversos fatores sociais como uso de drogas, álcool, usuários que residem sozinhos e os efeitos colaterais dos medicamentos. Portanto, é necessário que os profissionais consigam acolher esse usuário de forma adequada, com o intuído de envolve-lo na conduta terapêutica, diminuindo assim, os índices de abandono no tratamento.

Saber sobre o fluxo do atendimento aos usuários SR e também àqueles comprovados com TB, requer habi- lidades constantes e atualizadas, demonstrando haver discordância sobre o tempo que o diagnóstico demora para ficar pronto no laboratório de referência, sendo afirmado ser de dois a até 45 dias de espera. Esse é um dado importante no fluxo das ações, que as enfermeiras necessitam estar mais fundamentadas.

Mesmo havendo um caso de abandono no ano de 2016, foi possível identificar alguns aspectos importantes na conduta das enfermeiras, para com os usuários SR ou com TB, que necessitam de constantes aperfeiçoamentos e diálogo entre a equipe e usuários, assim como encontros com a coordenação, para estar continuamente construindo um fazer em saúde reflexivo, fundamentado e integral em prol da saúde coletiva.

Ressalta-se que o estudo não possui representatividade para SCS, pois não abrange todas as unidades de APS do município, mas é de grande importância para o aperfeiçoamento intelectual da pesquisadora e fonte de informações para o município e unidades de saúde alvo da pesquisa. 


\section{REFERÊNCIAS}

I. Ferri AO,Aguiar B,Wilhelm CM, Schmidt D, Fussieger F, Picoli SU. Diagnóstico da tuberculose: uma revisão. Revista Liberato [Internet]. 2014 [Acesso em 20 mai 20 I 7]; I5(24): I05202. Disponível em:http://www.liberato.com.br/sites/default/files/arquivos/Revista_SIER/v.\%2015,\%20n.\%2024\%20 (2014)/4\%20-\%20Tuberculose.pdf

2. Alves RS, Souza KMJ, Oliveira AAV, Palha PF, Nogueira JA, Sá L.D. Abandono do tratamento da tuberculose e integralidade da atenção na estratégia saúde da família. Texto contexto - enferm [Internet]. 2012 [Acesso em 24 nov 20 I7]; 2 I (3):650-657. Disponível em: http://www.scielo.br/scielo. php?script=sci_arttext\&pid=S0 I 04-070720 I 200030002 I

3. Brasil. Ministério da Saúde. Secretaria deVigilância em Saúde, Boletim Epidemiológico. v.46. n.9 Brasília: MS, 2015.

4. Sá LD, Souza KML, Nunes MG, Palha PF, Nogueira JA, Villa TCS. Tratamento da tuberculose em unidades de saúde da família: histórias de abandono. Texto contexto - enferm [Internet]. 2007 [Acesso em 10 abri 2017]; 16(4): 7I2-7I8. Disponível em: http://www.scielo.br/scielo.php?script=sci_arttext\&pid=SO 104-07072007004000 I 6\&lng=en. http:// dx.doi.org/I0.1590/S0 I04-070720070004000 I6.

5. BARDIN, L. Análise de conteúdo. São Paulo: Edições 70, 2011.279 p.

6. Daronco A, Borges TS, Sonda EC, Silveira CS, Bee GR, Passos PT. Aspectos relevantes sobre tuberculose para profissionais de saúde. Revista de Epidemiologia e Controle de Infecção, v.2, n.2, p.6I-65, Santa Cruz do Sul, 2012. Disponível em: <https://online.unisc.br/seer/index.php/epidemiologia/ article/view/2599/2063> Acesso em: maio de 2017.

7. Polaro SHI, Gonçalves LHT,Alvarez AM. Construindo o fazer gerontológico pelas enfermeiras das Unidades de Estratégia Saúde da Família. Rev Esc Enferm USP [Internet]. 2013 [Acesso em 20 nov 20I7]; 47(I):160-7. Disponível em: http://www.scielo.br/pdf/reeusp/v47n I/a20v47n I.pdf

8. Souza LRM, Oliveira LPBA, Medeiros ACQ, Menezes RMP. Ações de enfermagem no cuidado ao homem idoso na Estratégia de Saúde da Família. Rev enferm UFPE on line [Internet]. 2017 [Acesso em 20 nov 20I7]; I I(5):2024-32. Disponível em: http://www.revista.ufpe.br/revistaenfermagem/index.php/revista/article/view/8838/pdf_3199

9. Galavote HS, Zandonade E, Garcia ACP, Freitas PSS, Seidl H, Contarato PC, et al. O trabalho do enfermeiro na atenção primária à saúde. Esc Anna Nery [Internet]. 2016 [Acesso em 2 I nov 20I7]; 20(I):90-98. Disponível em: http://www. scielo.br/pdf/ean/v20n I/|4 |4-8I45-ean-20-0 I-0090.pdf

10. Macedo SM, Andrade RPS, Souza CBA, Andrade ASS, Villa TCS, Pinto ESG. Estratégias para capacitação ao cuidado em tuberculose. Cogitare Enferm [Internet]. 2016 [Acesso em 17 nov 20I7]; 2I(3):0I-08. Disponível em: http://revistas.ufpr.br/cogitare/article/view/45339

I I. Barrêto AJR, Evangelista ALF, Sá LD, Almeida SP, Nogueira JÁ, Lopes AMC. Gestão do cuidado à tuberculose: da for- mação à prática do enfermeiro. Rev. bras. enferm [Internet]. 2013 [acesso em 23 nov 2017]; 66(6):847-853. Disponível em: http://www.scielo.br/scielo.php?script=sci_arttext\&pi$d=$ S0034-7 I 6720 I 3000600006\&Ing=en.

12. Sousa GP, Baldoino LS, Silva MRS. Atuação do Enfermeiro no Controle da Tuberculose Pulmonar em Unidades Básicas de SaúdeTeresina-PI. Revista Interdisciplinar [Internet]. 2016 [acesso em 23 nov 2017]; 9(4):I22-13I. Disponível em: https://revistainterdisciplinar.uninovafapi.edu.br/index. php/revinter/article/view/997

13. Costa SM, Araújo FF, Martins LV, Nobre LLR, Araújo FM, Rodrigues CAQ. Agente Comunitário de Saúde: elemento nuclear das ações em saúde. Cien Saude Colet [Intenet]. 2013 [acesso em 23 nov 2017]; 18(7): 2147-2156. Disponível em: http://www.scielo.br/scielo.php?script=sci_arttext\&pid=S|4 | 3-8 | 2320 I 3000700030

14. Gomes RCM, Souza CD, Baggio L, Wachs F. O trabaIho do agente comunitário de saúde na perspectiva da educação popular em saúde: possibilidades e desafios. Ciênc. saúde coletiva [Internet]. 2016 [acesso em 23 nov 2017]; 2 I(5):I637-1646. Disponível em: http://www.scielo.br/scielo.php?script=sci_arttext\&pi$\mathrm{d}=\mathrm{S}|4| 3-8|2320| 600050|637 \&| \mathrm{ng}=$ en.

I5. BRASIL. Ministério da Saúde. Departamento de Vigilância Epidemiológica. Manual de recomendações para o controle da tuberculose no Brasil. I. ed. Brasília: Ministério da Saúde. 2011.284 p.

16. Caliari JS, Figueiredo RM. Tuberculose: perfil de doentes, fluxo de atendimento e opinião de enfermeiros. Acta Paul Enferm [Internet]. 2012 [acesso em 23 nov 20I7]; 25(I):4347. Disponível em: http://www2.unifesp.br/acta/pdf/v25/n I/ v25nla8.pdf

17. Araujo MRS, Silva HP, Silva AKLS. Avaliação situacional de biossegurança em tuberculose em Unidades Básicas de Saúde na Amazônia. Rev. bras. saúde ocup. [Internet]. 2016 [acesso em 23 nov 20I7]; 4I(2I). Disponível em: http://www.scielo.br/scielo.php?script=sci_arttext\&pi$d=$ S0303-765720 I 6000 I 002 I 2\&Ing=en

18. Ministério da Saúde. Secretaria de Vigilância em Saúde. Programa Nacional de Controle da Tuberculose. Guia de orientações para coleta do escarro. Brasília, 2014.

19. Chirinos NEC, Meirelles BHS, Bousfield ABS. A relação das representações sociais dos profissionais da saúde e das pessoas com tuberculose com o abandono do tratamento. Texto Contexto Enferm [Internet]. 2017 [acesso em 24 nov 20l7]; 26(I):e56500I5. Disponível em: http://www.scielo.br/pdf/tce/v26n I/pt_0I04-0707-tce-26-0 I-56500 I5.pdf

20. Rocha DS,Adorno RCF.Abandono ou Descontinuidade do Tratamento da Tuberculose em Rio Branco, Acre. Saúde Soc. São Paulo [Internet]. 2012 [acesso em 24 nov 20l7]; 2I(I):232-245. Disponível em: http://www.scielo.br/pdf/ sausoc/v2 In I/22.pdf 